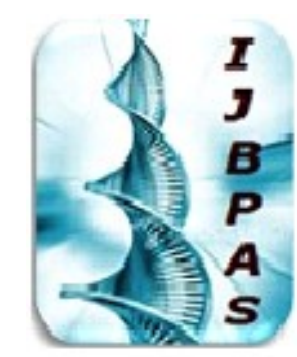

International Journal of Biology, Pharmacy and Allied Seiences (IJBPAS)

'A Bridge Betueen Caboratory and Q Qnendo'

\title{
WwW.ibpas.com
}

\section{USE OF GOMPHRENA GLOBOSA STAIN AS AN ALTERNATIVE TO EOSIN IN \\ ROUTINE HEMATOXYLIN AND EOSIN STAINING IN HISTOPATHOLOGY}

\section{UMASHANKAR K ${ }^{1^{*}}$, RAMANI $\mathrm{P}^{2}$, SHERLIN HJ ${ }^{2}$, GHEENA $\mathrm{S}^{3}$, RAMASUBRAMANIAM $A^{3}$, JAYARAJ $G^{3}$, DON KR ${ }^{3}$ AND SANTHANAM $A^{4}$}

1: Department of Oral and Maxillofacial Pathology, Saveetha Dental College \& Hospitals

Saveetha Institute of Medical \& Technical Sciences, SIMATS, Velapanchavadi, Chennai - 600077, India

2: Professor and Head, Department of Oral and Maxillofacial Pathology, Saveetha Dental College \& Hospitals, Saveetha Institute of Medical \& Technical Sciences, SIMATS, Velapanchavadi, Chennai 600077

3: Reader, Department of Oral and Maxillofacial Pathology, Saveetha Dental College \& Hospitals,

Saveetha Institute of Medical \& Technical Sciences

SIMATS, Velapanchavadi, Chennai - 600077

4: Senior Lecturer, Department of Oral and Maxillofacial Pathology, Saveetha Dental College \& Hospitals, Saveetha Institute of Medical \& Technical Sciences, SIMATS, Velapanchavadi, Chennai 600077

*Corresponding Author: Krishnapriya Umashankar: E Mail: Krishnapriva91@gmail.com

Received 25 ${ }^{\text {th }}$ Aug. 2020; Revised 14 ${ }^{\text {th }}$ Sept. 2020; Accepted $11^{\text {th }}$ Nov. 2020; Available online $1^{\text {st }}$ Aug. 2021

\section{https://doi.org/10.31032/IJBPAS/2021/10.8.5561}

\section{ABSTRACT}

Background Gomphrena globosa, is an edible plant from the family of Amaranthaceae. The flowers are rich in betacyanin and possess red violet colour. It has a stable $\mathrm{pH}$ ranging between 3 to 7 . To our knowledge, the current study represents the first initiative of using an extract of this flower in staining of oral tissue sections.

Aim This experimental comparative study aimed to explore the efficiency of Gomphrena globosa as natural staining dye for oral tissue histological sections as compared to the routinely and commonly used stain, hematoxylin and eosin. 
Materials and Methods Both fresh flower petals and dried flower petals were used for the process of preparation of the stain. Four different methods were used for the process of stain preparation. Fresh petals were grounded in mortar and pestle and this filtered and used. Dried flower petals were ground into fine powder. One portion of the fresh extract was boiled to $60^{\circ} \mathrm{C}$ and then this was then used staining purpose. $15 \mathrm{~g}$ of this powder was then mixed in $100 \mathrm{ml}$ of distilled water. This was then filtered and used. Finally the $2.5 \mathrm{~kg}$ of fine powder was mixed in $500 \mathrm{ml}$ of distilled water for 48 hours. This was then filtered. This solution was then placed in a Lyophilizer for 15 days at $-55^{\circ} \mathrm{C}$ for freeze drying. The viscous mixture thus obtained was used for the process staining.

Results The efficacy of the Globosa stain was nil when compared to routine H\&E staining.

Conclusion Though the pigment molecules present in Gomphrena globosa (Betacyanins) were same as compared to beetroot, the complexity in its chemical structure did not facilitate ionization, Thus the staining efficacy was nil.

\section{Keywords Betacyanins, Gomphrenin, Lyophilizer, Maceration}

\section{INTRODUCTION}

Stains in histopathology are substances or biological dyes which colour tissues in order to aid optical differentiation of tissue components [1]. Dyes are substances that impart colours to material such as textiles, cosmetics, foods, drugs, rubbers, plastics, hair, fur, and tissues [4]. Dyes are classified into synthetic and natural dyes [2].

Until the middle 19th century, all dyes were natural products extracted in mostly from plants and animals [3]. Haematoxylin was derived from Heamatoxylum campechianum, the dye derived from insect that is carmine from body of a female insect known as Dactilopius cacti; Orcein dye was from Crocus sativus [3]. Synthetic dyes consists of large group of organic compound derived from benzene (C6H6).
This consists of six carbon and hydrogen atoms [10]. Synthetic dyes are also known as coal tar since they manufactured from substance which were in the past only obtained from coal tar [3].

Hematoxylin-eosin stain is the most important routine histological stain that has been used in histopathology [4]. It is a combination of natural hematoxylin stain and synthetic eosin stain. Hematoxylin is a basic dye that stains acidic components of the cell, eosin is an acidic dye that stains the basic cytoplasmic components of the cells [4]. Nuclei of the cells take the hematoxylin dye and appear dark violet or blue in colour, cytoplasm of some epithelial cells, erythrocytes take eosin dye and stain pink [4]. 
Gomphrena globosa is an ornamental plant belonging to Amaranthaceae family of Caryophyllales order [5]. The simplest betacyanin, which was found in the petals of Gomphrenaglobosa is gomphrenin I (betanidin 6-O- $\beta$-glucoside) and its $15 \mathrm{~S}-$ diastereomer [6]. The majority of other Gomphrena globosa betacyanins are acylated by hydroxycinnamic acids (ferulic, p-coumaric, sinapic) derivatives of gomphrenin I [7].

This research work was done to explore the histological staining abilities of the dye extract of Gomphrena globosa (Globe amaranth). This experimental comparative study aimed to explore the efficiency of Gomphrena globosa as natural staining dye for oral tissue histological sections as compared to the routinely and commonly used stain, hematoxylin and eosin.

\section{MATERIALS AND METHODS}

An experimental comparative study was conducted on formalin fixed paraffin embedded blocks (Normal and pathological tissues) from archives of Department of Oral Pathology, Saveetha Dental College \& Research Institute, Chennai, Tamil Nadu, India. The study was conducted for a period of 1 month.

From these blocks, 12 of $4 \mu$ m-thick tissue sections were obtained and made into 4 equal groups. The first group of sections was stained by fresh extract of globosa, the second group by boiling method, the third group by maceration, and the fourth group by commercial extract. Each was divided in to 4 subgroups. Slides from each subgroup were stained for 10 minutes, 60 minutes and overnight. Additional 12 sections were obtained and stained by $H \& E$ stain as control. Note: Gomphrena solution replaced eosin in H\&E staining method as cytoplasmic stain. All staining procedures were in room temperature $\left(25-30^{\circ} \mathrm{C}\right)$.

Preparation of Extract The Gomphrena globosa flower petals were handpicked and shade dried for 7 days. This was then grounded into fine powder. Various solvents such as water, ethyl acetate, methanol, chloroform, and acetone were used for pigment extraction. Of all these solvents, water yielded the maximum colour. However ethyl acetate, methanol, chloroform, and acetone did not yield any colour. Hence water was used as a solvent for the purpose of pigment extraction. Fresh extraction, maceration, boiling, and lyophilization methods were employed to obtain its extracts.

Fresh Extract The fresh petals of the globosa flowers were ground using mortar and pestle. The filtrate obtained was filtered and then used to stain the tissues.

Boiled Extract The fresh extract of the globosa flower was heated to a temperature of $60^{\circ} \mathrm{C}$ to increase its concentration. This 
filtrate was filtered and then used to stain the tissue,

Maceration About $15 \mathrm{~g}$ of finely petal powder was dissolved in $100 \mathrm{ml}$ of distilled water. The preparation was left undisturbed for 48 hours. The filtrate obtained was filtered and then used to stain the tissues.

Lyophilization Nearly $2.5 \mathrm{~kg}$ of finely powdered flower was mixed in $500 \mathrm{ml}$ of water. This was then filtered. The filtrate was then placed in Lyophilizer at $-55^{\circ} \mathrm{C}$ for freeze drying. This was kept for nearly 15 days. The viscous concentrated mixture thus obtained was used for tissue staining. This was diluted in the concentration of 15 $\mathrm{mg} / \mathrm{ml}$ and this extract was used for staining.
Routine staining procedure of $H \& E$ was followed. Instead of eosin, globosa stain was substituted. To check for the staining ability, intensity and paucity of the of the globosa stain, 4 subgroups containing 3 tissue sections were left in the globosa stain for 10 minutes, 30 minutes and overnight and the results were interpreted as intense, scares and nil staining.

With fresh solution, boiled solution, maceration and lyophilized extract, nil results were noted at 10 minutes, 30 minutes and overnight staining process (Table 1). The tissue samples did not take up the stain and were noted to be colourless. Hence the results obtained were noted to be nil.

\section{RESULT}

Table 1: Staining efficacy of different extracts of globosa pigments

\begin{tabular}{|c|c|c|c|}
\hline Sample & $\mathbf{1 0}$ minutes & $\mathbf{3 0}$ minutes & Overnight \\
\hline Fresh extract & Nil & Nil & Nil \\
\hline Boiled extract & Nil & Nil & Nil \\
\hline Maceration & Nil & Nil & Nil \\
\hline Lyophilized extract & Nil & Nil & Nil \\
\hline
\end{tabular}

\section{DISCUSSION}

During staining, dye molecules in stain appear as a certain colour and attached to a specific site or cellular structure [9]. Combination of stains may be necessary to affect certain tissue demonstrated [9]. This goes to show that a dye must ionize in solution to produce coloured cations and anions which are capable of uniting with tissue components to form coloured compounds [9].
There are various factors which affect the tissue dye affinities [10]. These factors include coulombic attractions, Van der Waal's forces, hydrogen bonding, covalent bonding. The solvent-solvent interactions include hydrophobic effect [10]. The affinity of the dye towards the tissues describes the tendency of a stain to transfer from a solution onto a section [10]. Moreover the uptake of dyes and reagents is often multistep, in both space and time 
[10]. Gomphrena globosa flower extract with aqueous extraction in this research has shown nilstaining quality.

Gomphrena globosa is an ornamental plant belonging to Amaranthaceae family of Caryophyllales order [11]. Within the Gomphrena genus, there are several varieties of these plant species [11]. Previous studies proved that the purple petals of their inflorescences contain betacyanin pigments. The first effort in examination of composition of betacyanins in Gomphrena globosa inflorescences was conducted in 1967 [11]. The more recent studies proved occurrence of much more varieties of betacyanins [11]. The simplest betacyanin, which was found in the petals of Gomphrenaglobosa is gomphrenin I (betanidin 6-O- $\beta$-glucoside) and its $15 \mathrm{~S}$ diastereomer (Figure 1) [11, 12, 13]. The majority of other Gomphrena globosa betacyanins are acylated by hydroxycinnamic acids (ferulic, pcoumaric, sinapic) derivatives of gomphrenin I. The base difficulty of study on these compounds and their afterward exploration is isolation of selected pigments with high purity with efficient purification.

Thus keeping all these factors in mind, though the $\mathrm{pH}$ of the extract was acidic, the other factors such as the affinity, ionization capacity did not attribute to the staining effect thereby giving nil staining of the cellular components [10]. Though, Sherin et al in 2017 have given positive staining by betacyanins of the beetroot extracts in oral smears, the complex chemical structure of betacyanins in the Gomphrena globosa would not have not facilitated the ionization thus exhibiting nil staining of the tissue components [5]. Thus this could not be used as an alternative to eosin in routine $\mathrm{H} \& \mathrm{E}$ in histopathological staining.

\section{CONCLUSION}

As a conclusion of this study, Gomphrena globosa solution cannot be used as a cytoplasmic stain instead of eosin in H\&E, method to stain formalin fixed paraffinembedded tissue sections. Thus variations in chemical structure of organic pigments in natural substances also attribute to the staining ability of the natural stains.

\section{REFERENCES}

[1] Sunday Ewaoche Itodo, Steve Oyero, EU Umeh, Amodu Ben and Musa Dauda Etubi. Phytochemical properties and staining ability of Red Onion (Allium cepa) extract on histological sections. J. Cytol. Histol: 2014; 5: 6-8.

[2] Sherin Singnarpi, Pratibha Ramani, AnujaNatesan, Herald J Sherlin, Gheena S, Giffrina, Abhilasha, Don, and Archana. Vegetable Stain as an Alternative to H\&E in Exfoliative Cytology. J CytolHistol: 2017; 8: 3-6. 
[3] Marin Abraham, Shwetha Nambiar, Vanishri Charagannavar, Dominic Augustine, SV Sowmya, Ashok babu, Roopa s rao. Comparison of Staining Efficacy between Turmeric and Eosin: A Histological Study. Journal of Clinical and Diagnostic Research. 2017 Nov; Vol-11(11): ZC05-ZC08; 1-5.

[4] Esam M. A. Raheem, Abd-Alhafeez Osman Ibnouf, Osman Hashim S hingeray, Hamza JM Farah. Using of Hibiscus Sabdariffa extract as a natural histological stain of the Skin. USA journals, 2015; 3(5): 1-2.

[5] Mousumi Biswas, Satyahari Dey, Ramkrishna Sen. Betalains from Amaranthustricolor. Betalains from Amaranthustricolor L. Journal of Pharmacognosy and Phytochemistry. Volume 1: Issue 5; 1-5.

[6] Himanshu Paliwal, Sachin Goyal, Shivali Singla, Shashi Daksh. Pharmacy science journal. 2016: Volume 1(3); 1-12.

[7] Nkechi Augustina Olise, Ifeoma Bessie Enweani, Ifeanyi Onyema Oshim, Evelyn Ukamaka Urama, Clinton Chinedu Ngwoke. The Use of Extracts from Nigerian Indigenous Plants as Staining Techniques in Bacteriology, Mycology and Histopathology. International Journal of Plant Research. 2018: 8(1); 15-24.

[8] Sudheendra Udyavara Sridhara, Shashidara Raju, Aparna H. Gopalkrishna, Vanishri C.
Haragannavar, D. Latha, Reshma Mirshad. Hibiscus: A different hue in histopathology. Journal of Medicine, Radiology, Pathology \& Surgery: (2016); 2, 9-11.

[9] Culling CFA (1974); Handbook of Histological and Histochemical techniques. 3rd ed. Butherworths Co. Ltd London.; 201-205

[10] John D. Bancroft, Christopher Layton, S Kim Suvarana. Bancroft's Theory and Practice of Histological Techniques. Seventh edition. Churchill Livingston. Elsevier; 158160.

[11] Dominika Szot, Karolina Starzak, Anna Skopińska, Sławomir Wybraniec. Chromatographic fractionation of betacyanins from flowers of Gomphrena globosa. Journal of chromatography A. 2015. Vol 6 (2); 1-5.

[12] L. Minale. Pigments of Centrospermae - VII. Betacyanins from Gomphrena Globosa. Phytochemistry, 1967: 6; 703-709,

[13] S. Heuter, Betacyanins from flowers of Gomphrena Globosa. Phytochemistry. 1992 31(5); 18011807. 